\title{
Regulation of carotenoid biosynthesis genes during plant development
}

\author{
Pablo A. Scolnik a and Giovanni Giulianob \\ a DuPont Central Research, P. 0. Box 80402, Wilmington, \\ Delaware 19880-0402, USA, and b Ente Per Le Nuove Tecnologie, \\ l'Energia e l'Ambiente, PO Box 2400, Rome 00100 AD, Italy.
}

\begin{abstract}
Expression of the carotenoid biosynthesis genes $P D S$ and $P S Y$ during development of tomato plants was studied by reverse transcriptase-polymerase chain reaction ( $R T-P C R$ ). Results indicate that both stress and developmental signals control the expression of these genes. The $P D S$ was mapped to the chromosome 3 of tomato.
\end{abstract}

\section{INTRODUCTION}

Plant genes for enzymes of the carotenoid biosynthesis pathway are nuclear, and the protein products are imported into organelles. Molecular probes for carotenoid biosynthesis genes are now becoming available. In the last year $P D S$, encoding phytoene desaturase, has been cloned from soybean (ref. 1), pepper (ref. 2), and tomato (G. E. Bartley and P. A. Scolnik, unpublished, Genbank accession number M88683; Pecker et al., 1992), and PSY, encoding phytoene synthase, was identified as corresponding to pTOM5, a CDNA for a ripeninginduced tomato gene (Refs. 4, 5).

\section{GENE EXPRESSION}

We are using tomato as a model system to study the developmental aspects of carotenoid biosynthesis in plants. The low level of expression of $P D S$ and $P S Y$ precluded studies in organs other than fruits. We have now used a sensitive mRNA detection method to study the expression of $P S Y$ and $P D S$ during the development of tomato plants. The reverse transcriptase-polymerase chain reaction (RTPCR amplification assay provides the level of sensitivity required to study rare transcripts (Fig. 1). To study PSY and PDS expression by RT-PCR, we used reverse transcriptase and RNA isolated from different tomato organs to produce first-strand CDNA. We then amplified the PDS and PSY CDNAs with Taq polymerase, and we measured the products by ethidium bromide-induced fluorescence. Using RT-PCR we were able to detect expression of both genes in all organs of mature tomato plants. The signals from the corresponding transcripts vary significantly in the different samples, indicating control of gene expression by light and development.

The levels of PDS and PSY transcripts were determined in etiolated seedlings exposed to light and in light-grown seedlings transferred to darkness. Results indicate that $P D S$ mRNA levels vary less than 2-fold during either de-etiolation or dark adaptation. PSY mRNA 
levels also remain essentially constant during de-etiolation, but they decrease about 7-fold upon dark adaptation (Fig. 2). Thus, expression of $P D S$ and $P S Y$ is essentially constitutive during photomorphogenesis. After photomorphogenesis is complete, the only gene which seems to be under light control is PSY. Colored carotenoids are required for chloroplast differentiation. Therefore, a block in their synthesis results in arrested chloroplast development, which in turn results in lower mRNA levels for several nuclear genes that encode chloroplast proteins (reviewed in ref.6).

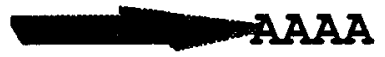

I. Isolation of RNA

Primer 1

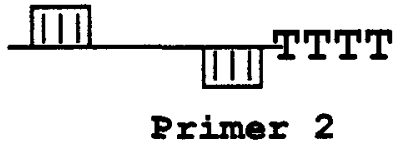

III. RCR exponential amplification

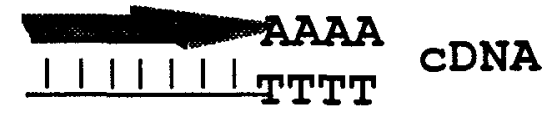

II. Reverse transcription

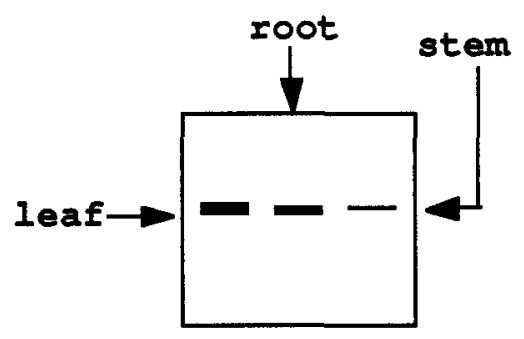

IV. Agarose Gel

Eig. 1. Reverse transcriptase-polymerase chain reaction (RT-PCR): sensitive detection of rare mRNAs.

De-etiolation

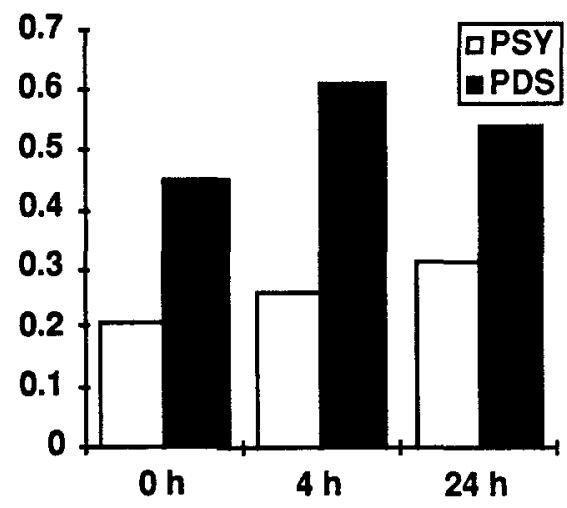

Dark Adaptation

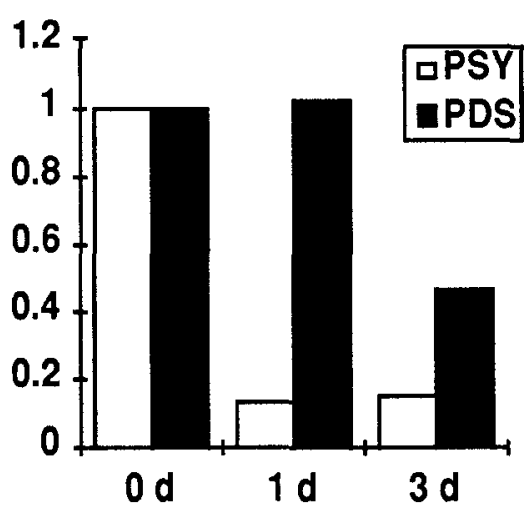

Eig. 2. Light regulation of $P S Y$ and $P D S$ expression.

Since carotenoid genes are nuclear genes encoding chloroplast proteins, their expression was investigated in seedlings of the ghost mutant, which blocks the desaturation of phytoene, or wildtype seedlings treated with the Pds inhibitor Norflurazon. Seedlings that lack colored carotenoids contain increased mRNA levels for PSY (2-3 fold) and PDS (5-10 fold) (Eig. 3). 
Thus, blocks in carotenoid biosynthesis induced by Norflurazon treatment or by the ghost mutation, induce PDS expression and, to a lesser extent, PSY expression. This induction could be due either to photooxidative stress or, as in fungi (Ref. 7), to feedback regulation of carotenogenesis. Current unpublished experiments indicate that $P D S \mathrm{mRNA}$ induction requires both an arrest in carotenoid biosynthesis and light, suggesting that this gene responds to photo-oxidation.

$P S Y$ and $P D S$ expression were analyzed during leaf, flower, and fruit development. Between leaf stage 1 (approximate length $35 \mathrm{~mm}$ ) and stage 4 (approximate length $140 \mathrm{~mm}$ ), PSY mRNA levels decline slightly, whereas $P D S$ expression increases more than 3-fold (not shown, ref. 8).

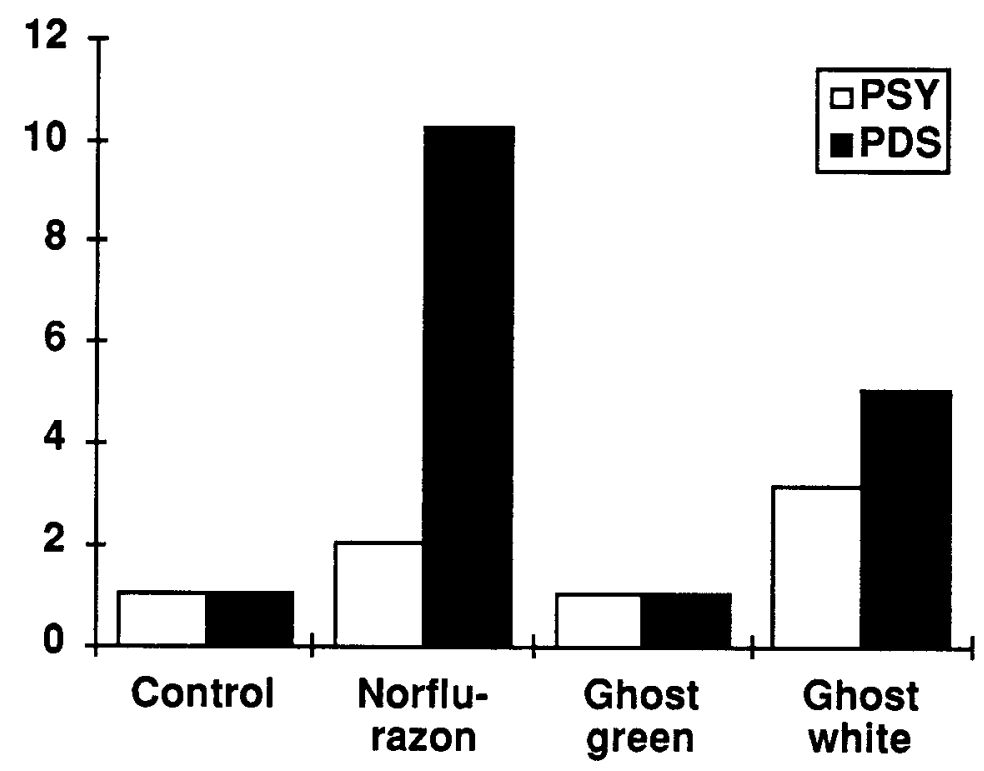

Eig. 3. PSY and PDS expression in seedlings.

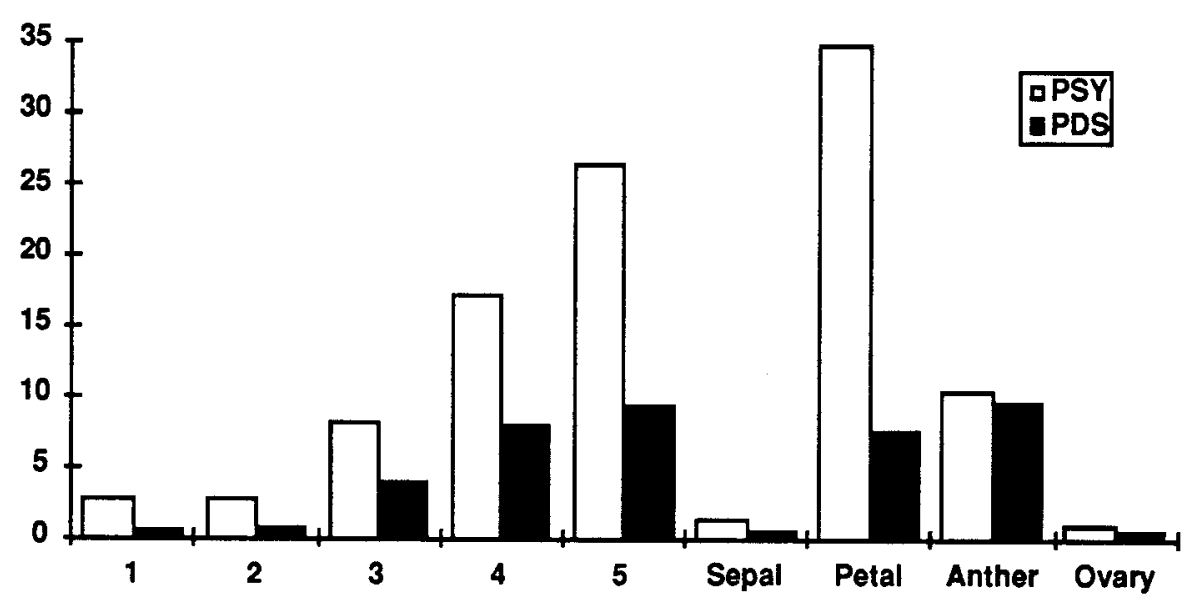

Eig, 4. PSY and PDS expression in flower. 
In early stages of flower development PSY expression is slightly higher than in mature leaves, whereas PDS is expressed at basal levels. Expression of both genes is induced approximately 10-fold during flower development (Fig. 4). Dissection of flowers into the four organ whorls indicates that this induction is due to high expression levels in chromoplast-containing (petals and anthers) organs, while basal levels of expression are maintained in sepals and ovaries.

We measured expression of $P D S$ and $P S Y$ during fruit ripening, when the color of the pericarp changes from green to red as chlorophyll is degraded and carotenoids accumulate. During this process chloroplasts differentiate into chromoplasts, chlorophyll content declines to undetectable levels, and carotenoid content increases. From immature green to orange, $P S Y$ is induced $>25$-fold but $P D S$ mRNA levels increase less than 3-fold (Fig. 5).

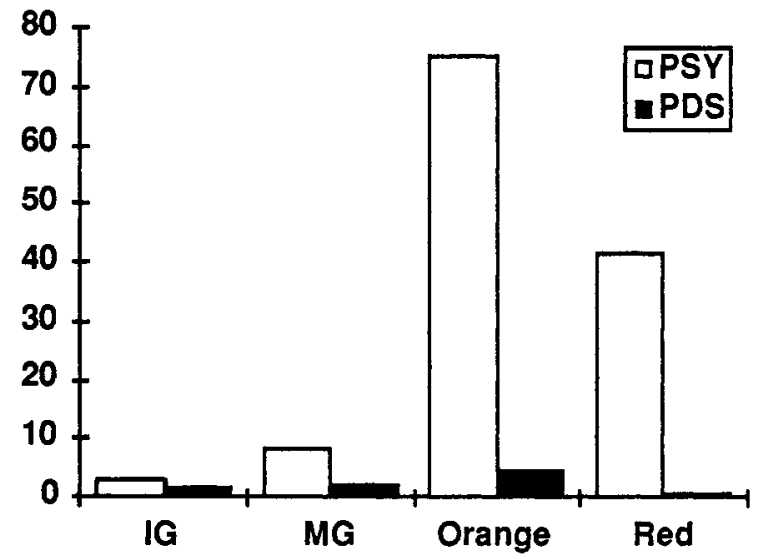

Eig. 5. PSY and PDS expression in Fruit. Green stages: immature (IG) and mature (MG).

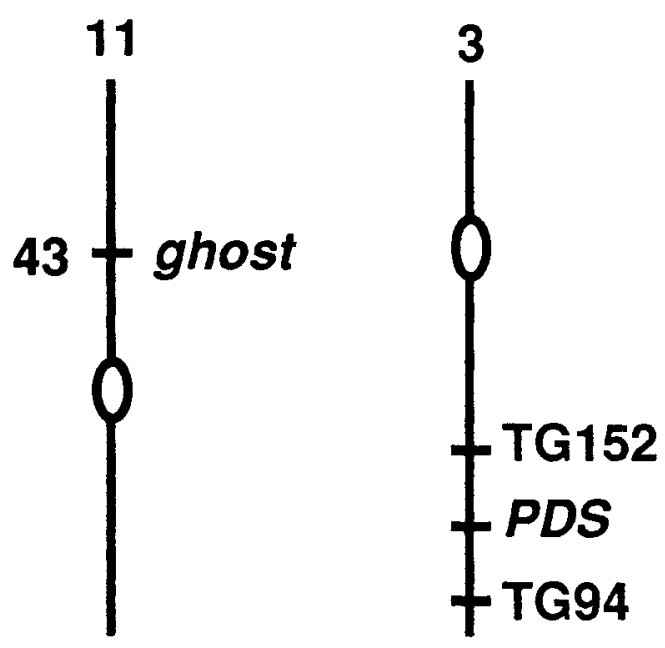

Eig. 6. Genome mapping of PDS and ghost. 
PSY and PDS are maximally expressed in flowers and fruits and at very low levels in roots. Transcript levels in petals are higher than in sepals. Levels of mRNA in anthers are also higher than in sepals for both PDS (>17-fold) and PSY (>7-fold). The difference in mRNA levels between petals and roots is $>316$-fold for $P S Y$ and $>94-$ fold for PDS. Expression of both genes in stems is slightly lower than in mature leaves.

\section{MAPPING}

To determine whether the ghost mutant of tomato corresponds to a lesion in PDS, we mapped this gene in the tomato genome. An XbaI polymorphism present in this gene between Lycopersicon esculentum and $L$. penelii was scored in a population of $67 \mathrm{~F} 2$ individuals from a $L$. esculentum $\times L$. penelii cross (K. Alpert, personal communication). Segregation of the corresponding alleles showed no significant deviation from the expected 1:2:1 ratio $\left(\chi^{2}=2.16\right)$. According to the linkage data, PDS maps to chromosome 3 , at approximately 10.2 and 14.5 centimorgans from markers TG94 and TG152, respectively (Fig. 6). Therefore, this gene does not correspond to the GHOST locus, which maps to chromosome 11.

\section{CONCLUSIONS}

By using RT-PCR we were able to demonstrate regulation of PSY and PDS by flower and fruit development and by photooxidation. We have also shown that expression of both genes is not affected by deetiolation, a process that results in a 4-fold increase in carotenoid levels. Analysis of PSY and PDS promoters is in progress to elucidate the molecular basis of these control mechanisms.

\section{ACKONOWLEDGEMENTS}

Work in the laboratory of G.G. was supported by grants from the Italian Ministry of Agriculture and by the European Community (projects BRIDGE and BIOTECH). We acknowledge the excellent genome mapping work of Kevin Alpert (Campbell Research Institute, Davis, CA).

\section{RHFERENCES}

1. G. E. Bartley, S. A. Coomber, D. M. Bartholomew, and P. A. Scolnik. In cell culture and somatic cell Genetics of plants, vel. 7B, (I. Bogorad and I. K. Vasil, eds) pp. 331-364 Academic Press, New York (1991a).

2. P. Hugueney, S. Römer, M. Kuntz, and B. Camara. Eur. J. Biochem. 209, 399-407 (1992).

3. I. Pecker, D. Chamovitz, H. Linden, G. Sandmann, and J. Hirschberg. Proc. Nat1. Acad. Sci. USA 89, 4962-4966 (1992).

4. G. E. Bartley, P. V. Viitanen, K. O. Bacot, and P. A. Scolnik. J. Biol. Chem, 267, 5036-5039 (1992). 
5. P. Bramley, C. Teulieres, I. Blain, C. Bird, and W. Schuch. Blant I. 2, 343-349 (1992).

6. W. C. Taylor. Annu. Rev. Plant Physiol. Plant Mol. Biol, 40, 211-233 (1989).

7. E. R. Bejarano, and E. Cerdá-Olmedo. Phytochemistry 28, 1623-1626 (1989).

8. G. Giuliano, G. E. Bartley, and P. A. Scolnik. Plant Cell 5, 379387 (1993). 